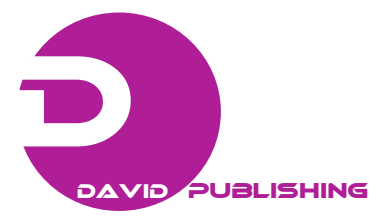

\title{
Modeling and Simulation of Hydraulic Long Transmission Line by Bond Graph
}

\author{
Dragan Pršić, Novak Nedić and Ljubiša Dubonjić \\ Faculty of Mechanical Engineering Kraljevo, University of Kragujevac, Kraljevo 36 000, Serbia
}

Received: February 6, 2013 / Accepted: March 7, 2013 / Published: April 25, 2013.

\begin{abstract}
This paper addresses the issue of modeling of the hydraulic long transmission line. In its base, such model is nonlinear with distributed parameters. Since general solution in closed-form for such model in time-domain is not available, certain simplifications have to be introduced. The pipeline in the paper has been divided to a cascaded network of $\pi$ segments so that a model with lumped parameters could be reached. For segment modeling, a standard library of bond graphs element has been used. On the basis of models with lumped parameters, the effect of the number of segments, pipeline length and effective bulk modulus on the dynamics of long transmission line have been analyzed.
\end{abstract}

Key words: Long transmission line, lumped parameters, $\pi$ segments, bond graph.

\section{Introduction}

Existence of a long transmission line (LTL) in hydraulic systems makes their dynamics significantly complex. This is especially emphasized with building and mining machines, agricultural machines, transportation machines, machine tools and other devices where connection between the actuators energy source achieved by a long hydraulic line. Physical variables, pressure and volumetric flow, featuring the energy transfer along the hydraulic line, besides the time coordinate, depend on spatial coordinate as well. These physical variables' dependency on spatial coordinate conditions spatial distribution cannot be neglected in long hydraulic line modeling. Therefore they are described with models with distributed parameters. Models with distributed parameters are described with partial differential equations. Use of such a model in analysis of dynamic behavior in time domain is not practical because it requires work with transcendent transfer functions and their

Corresponding author: Dragan Pršić, assistant professor, Ph.D., research field: modeling and simulation. E-mail: prsic.d@mfkv.rs. approximations using Bessel functions.

In this paper, we have used the method of description of a long hydraulic pipe with lumped parameters in a way that it was divided to $n$ equal segments of $L s$ length. $\pi$ and $T$ model with lumped parameters have been used whose electrical analogies are given in the paper. On the basis of equivalent electric circuits, adequate bond graphs of these circuits were made and connected into a cascade of $n$ segments, which defines the mathematical model of the hydraulic pipe. On the basis of the mathematical model and simulation, we have analyzed the impact of certain parameters on the character of the transfer process and its results are given in the paper.

The paper is organized as follows: Section 2 starts from the description of one-dimensional flow of compressible viscous fluid through LTL. Approximation of this model is then introduced through a cascade of small lumped elements described by means of common differential equations. For each element, there is a corresponding electric circuit, i.e., a bond graph model. Based on the models from section 2, section 3 gives simulation results for LTL with input flow as an excitation variable and a hydraulic orifice at 
the end of the line. Conclusions are outlined in section 4 .

\section{Modeling of Hydraulic Long Transmission Line}

One-dimensional flow of compressible, viscous fluid through the LTL is represented by a set of nonlinear partial differential equations [1]. Applying physical principles of mass conservation, Newton's second law and energy conservation leads to

$$
\begin{gathered}
\frac{A}{a^{2}} \frac{\partial p}{\partial t}=-\rho \frac{\partial Q}{\partial x} \\
\frac{1}{A} \frac{\partial Q}{\partial t}+g \sin \alpha+\frac{\lambda}{2 d A^{2}} Q^{2}=-\rho \frac{\partial p}{\partial x}
\end{gathered}
$$

That is a pair of quasi-linear hyperbolic partial differential equations describing pressure change $p$ and volumetric flow rate $Q$ depending on time $t$ and distance $x$ along the pipeline. Generally, there is no closed-form solution for these equations. The problem is particularly expressed in case of turbulent flow which introduces stochastic parameters. Models with distributed parameters are described by partial differential equations and, in the complex domain, these models are of infinitesimally high order [2-5].

In order to find analytical or numerical solution, certain simplifications have to be introduced. One approach is based on division of non-homogenous fields $(P(x, t), Q(x, t))$ on segments with homogenous fields $(P(t), Q(t))$ of all physical variables. In other words, instead of models with distributed parameters we move to the model with lumped parameters. Exactly this is the approach used in this study. LTL is observed as a cascaded network of small lumped elements (Fig. 1) where dynamics of each of them is described by common differential equations.

Each of lumped elements presents spatial abstractions of distributed physical properties. Physical dimensions of each segment are much smaller than the shortest wavelength of interest. Connections between lumped elements represent physical constraints on the physical quantities associated to the elements [6]. Practically, we need to do a model of one segment and

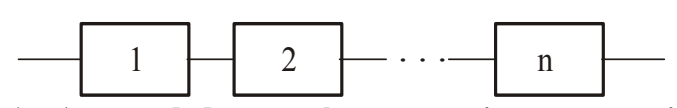

Fig. 1 A cascaded network representing a transmission line.

then serially connect those models into the system model (LTL model).

What we usually find in literature are two single-lump approximations for a short fluid line [7-8]. Those two approximations are known as $\pi$ and $T$ circuits. Their electrical analogies are given in Fig. 2.

Same elements for modeling of the lumped elements were used in both models. Using capacitor ( $C$ element), we model fluid compressibility, using coil ( $L$ element) we model fluid inertia, and with resistor ( $R$ element) we model losses due to friction. Difference is in the way those elements are connected. In the first case, there are two distinctive pressures: $P_{1}$ on one end and $P_{2}$ on the other end of the segment. Same flow rate $Q_{i}$ is adopted along the whole segment length. In the second case, there is one distinctive pressure of $P_{i}$ segment and two flows $Q_{1}$ and $Q_{2}$ on the segment's ends.

Which model is to be used depends on the remaining part of the circuit where the given pipeline is located. Namely, each segment is connected with the surrounding with two energy flows. On one end, the energy flow is $P_{1} Q_{1}$, and on the other $P_{2} Q_{2}$. This means that interaction of segments with the environment can be described with four variables. In regard to the segment, two values must be independent, and the other two dependent, provided that the independent values must be on different segment ends. For example, it is not allowed for the values $P_{1}$ and $Q_{1}$ to be independent. Therefore, it is possible to make four combinations of independent-dependent variables: $Q_{1} Q_{2} \rightarrow P_{1} P_{2}, \quad Q_{1} P_{2} \rightarrow Q_{2} P_{1}, \quad P_{1} Q_{2} \rightarrow Q_{1} P_{2} \quad$ and $P_{1} P_{2} \rightarrow Q_{1} Q_{2}$. Model of the segment, i.e. the whole pipeline, depends on the combination appearing in the circuit. It should be noted that when the segment cascade is made, regardless if we use $\pi$ or $T$ model, there are blocks inside the model shown in Fig. 3 that periodically repeat. 


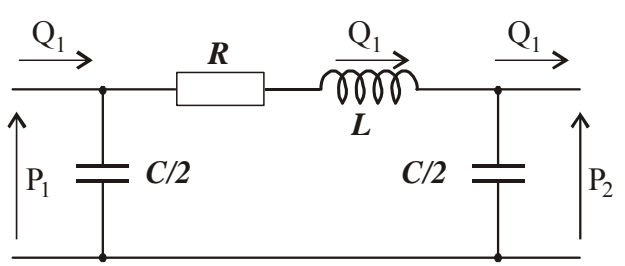

(a) $\pi$ circuit

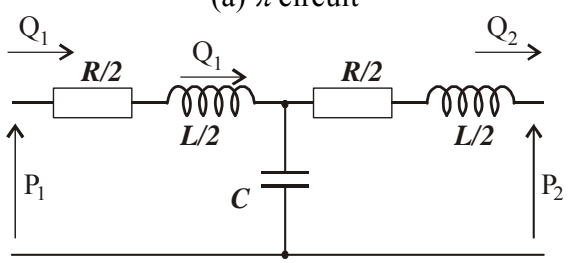

(b) $T$ circuit

Fig. 2 Equivalent electrical analogy of short fluid line.

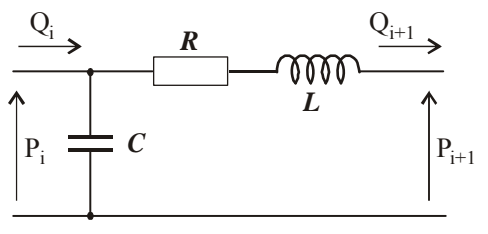

$\mathrm{i}=1,2,3 \ldots$

Fig. 3 Block of LTL model repeating periodically.

Difference in models comprising of $\pi$ and $T$ segments is in the end blocks - interfaces according to the remaining of systems symmetric in regard to internal blocks. Equivalent electrical circuits of end blocks of the cascade with $\pi$ segments ( $\pi$ interfaces) are shown in Fig. 4 and cascades with $T$ segments ( $T$ interfaces) in Fig. 5.

Suitable bond graphs of the electric circuits in Figs. 3-5 are shown in Figs. 6-8 respectively.

On the basis of drawn causality it can be seen that all internal blocks are second rate models. The end blocks for the $\pi$ cascade have integral causality for one-junction and arbitrary causality for zero-junctions. It is similar with the $T$ cascade where zero-junction has integral causality and one-junctions the arbitrary one.

This means that the LTL model can have minimum order of $(n-1) * 2+1$ and maximum $(n-1) * 2+3$. For example, cascade of $n=5$ segments can be minimally 9 order and maximum 11. Order of the model depends on the hydraulic circuit where the given LTL is found. In order to avoid the differential causality and reduce the numerical problem, recommendation is to use the $\pi$
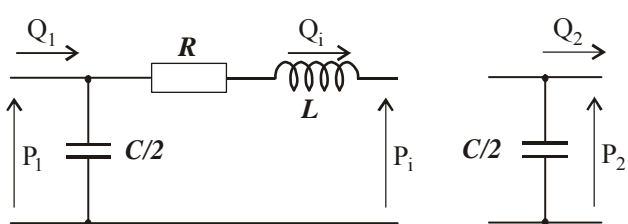

Fig. 4 LTL model interfaces with $\pi$ segments $(\pi$ interfaces).
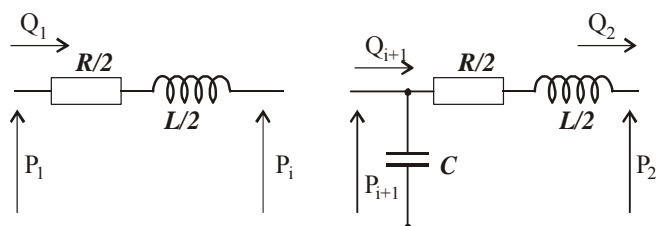

Fig. 5 LTL model interfaces with $T$ segments (T interfaces).

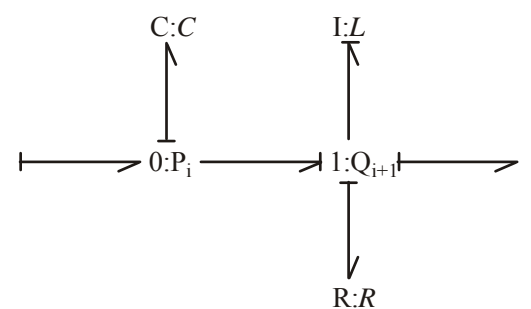

Fig. 6 LTL model bond graph repeating periodically.

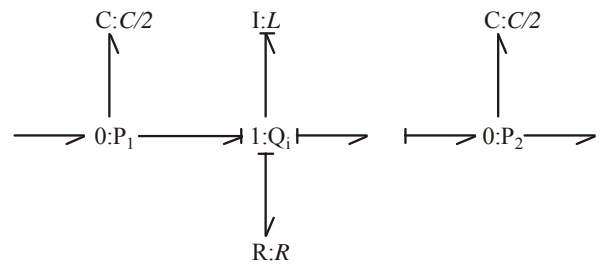

Fig. 7 LTL model interface bond graph with $\pi$ segments ( $\pi$ interfaces).

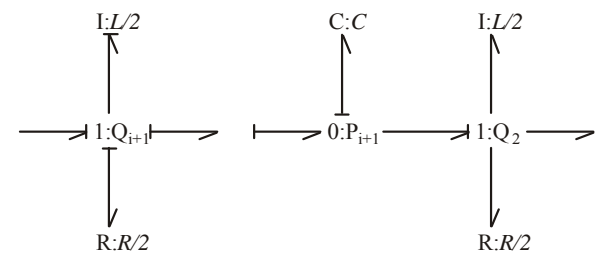

Fig. 8 LTL model interface bond graph with $T$ segments ( $T$ interfaces).

cascade if the flow is independent value. If pressure is independent value, differential causality is avoided with use of the $T$ cascade.

In this study, we observe the response of the system on the independent flow change. Diagram of hydraulic installation is shown in Fig. 9.

As a source of excitation, variable flow rate source $\left(Q_{1}\right)$ is used. LTL is represented as a cascade of $n$ segments. At the end of the circuit there is a hydraulic 


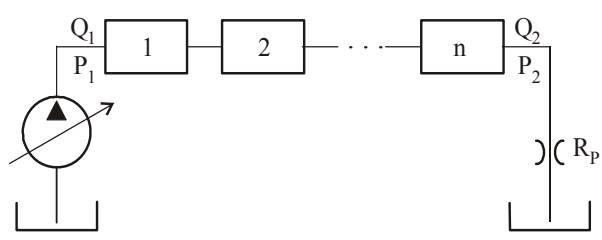

Fig. 9 Schematic of a circuit for LTL simulation.

orifice of constant resistance. Model order is determined with $2 n+1$.

\section{Simulation Analysis}

On the basis of $\pi$ model with interface shown in Fig. 4, behavior of LTL in circuit shown in Fig. 9 is simulated. The aim is to analyze the impact of several parameters on the transfer process.

The parameters at which the simulation was performed are $B_{e}=1.4 \times 10^{9} \mathrm{~Pa}, Q_{\text {ref }}=3.5 \times 10^{-4} \mathrm{~m}^{3} / \mathrm{s}$, $d=1 \times 10^{-2} \mathrm{~m}, \rho=860 \mathrm{~kg} / \mathrm{m}^{3}, v=9.7 \times 10^{-5} \mathrm{~m}^{2} / \mathrm{s}$.

Before the beginning of simulation, we need to define initial conditions for each segment's flow and pressure. It is assumed that volumetric flow rate and pressures are in the steady-state regime before the beginning of the transition process.

Figs. 10-12 present relative changes of the flow $Q_{2}$ and the pressures $P_{1}$ and $P_{2}$ for step increase of the flow $Q_{1}$ and for the pipeline of the same length $(L=16 \mathrm{~m})$. Three cases were observed: (a) $n=1, L s=16 \mathrm{~m}$; (b) $n$ $=4, L s=4 \mathrm{~m}$ and (c) $n=16, L s=1 \mathrm{~m}(n \times L s=$ const. $)$. The results show the influence of the number of segments on dynamics.

In all three cases the transition process has the character of damped oscillations. However, with the increase of the number of segments the basic harmonics of each value $\left(Q_{2}, P_{1}, P_{2}\right)$ are superposed with the harmonics of higher frequency. The larger number of segments, the higher frequencies in the frequency spectrum. These frequencies may lead to undesirable oscillations in the system [9].

Besides, delay in relation to the excitation value can be clearly noticed in the response of values at the other end of the pipeline $\left(Q_{2}, P_{2}\right)$.

The following Figs. 13-15 show impact of the pipeline length on transfer processes in LTL. In all three

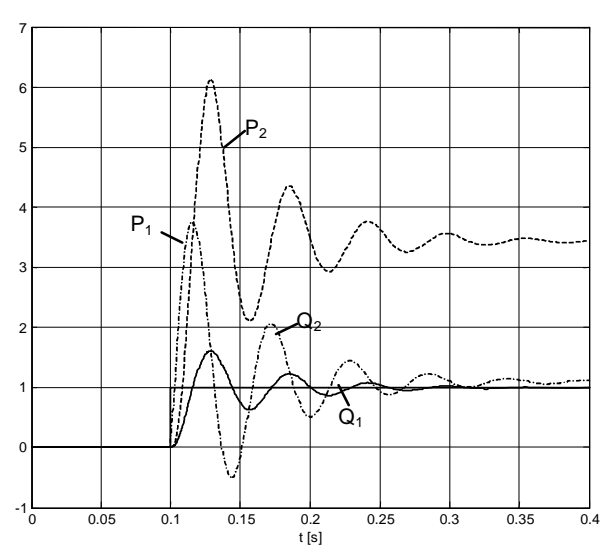

Fig. 10 Transient processes for $n=1$ and $L s=16 \mathrm{~m}$.

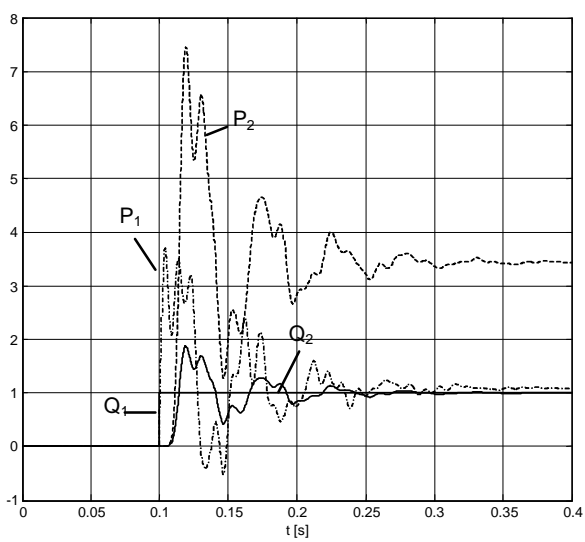

Fig. 11 Transient processes for $n=4$ and $L s=4 \mathrm{~m}$.

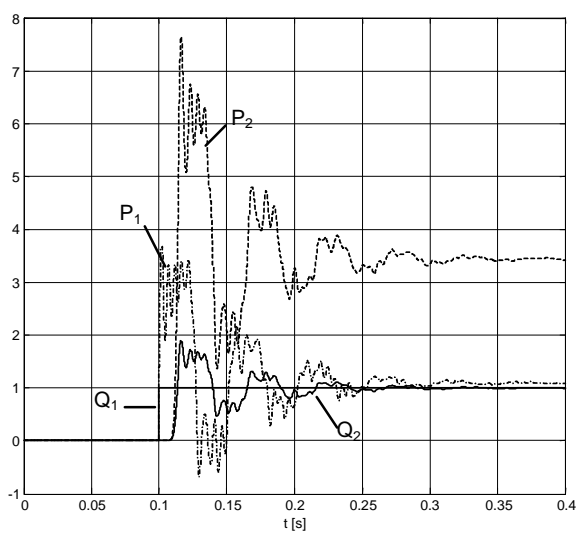

Fig. 12 Transient processes for $n=8$ and $L s=2 \mathrm{~m}$.

cases, $\pi$ segments of same length $L s=2 \mathrm{~m}$ are used for modeling. Difference is only in the number of segments.

The inertia of the system is increased with the increase of the pipeline length. The rise time and frequency of oscillations decline, whereas the damping ratio and delay increase. Particularly noted is reduction of magnitude on pressure $P_{2}$. 


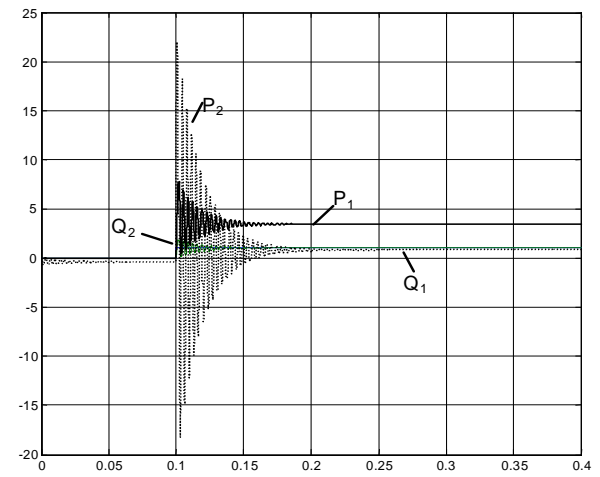

Fig. 13 Transient processes for $n=1$ and $L s=2 \mathrm{~m}$.

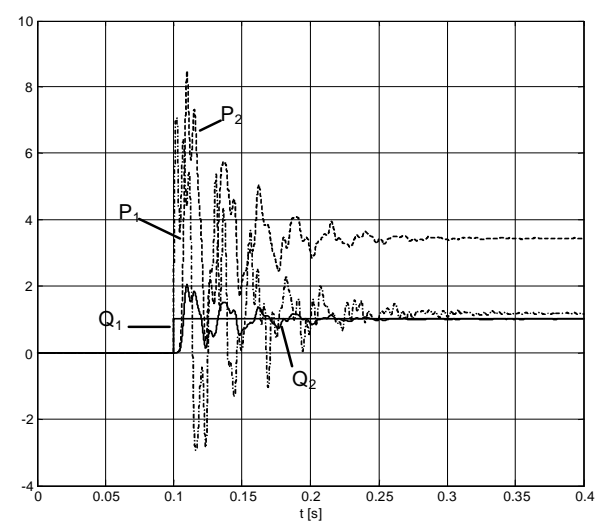

Fig. 14 Transient processes for $n=4$ and $L s=2 \mathrm{~m}$.

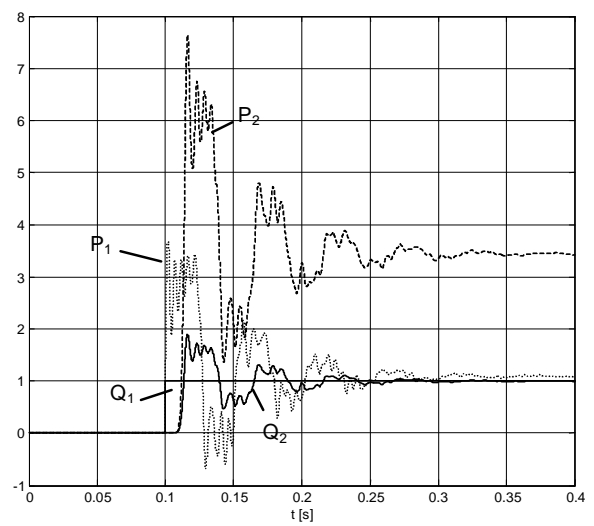

Fig. 15 Transient processes for $n=8$ and $L s=2 \mathbf{m}$.

The last parameter which influence on the transfer process we are analyzing is effective bulk modulus. In previous examples, we assumed that the elasticity of hydraulic pipe wall can be neglected. We also assumed that there is no entrapped vapor or gas in the fluid. Effective bulk modulus can be approximately determined using the expression [9]:

$$
\frac{1}{B_{e}}=\frac{1}{B_{C}}+\frac{1}{B_{l}}+\frac{V_{g}}{V_{t}} \frac{1}{B_{g}}
$$

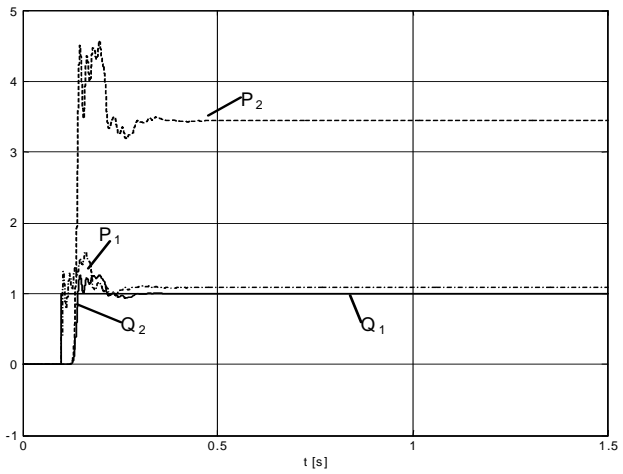

Fig. 16 Transient processes for $V_{g} / V_{t}=\mathbf{0 . 0 0 1}$.

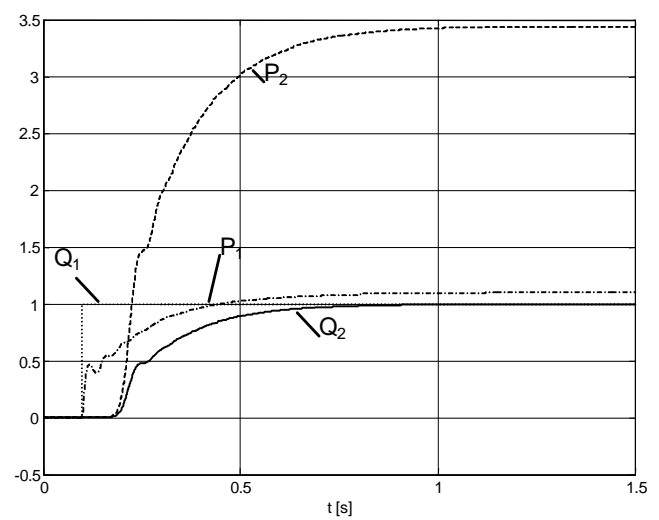

Fig. 17 Transient processes for $V_{g} / V_{t}=\mathbf{0 . 0 1}$.

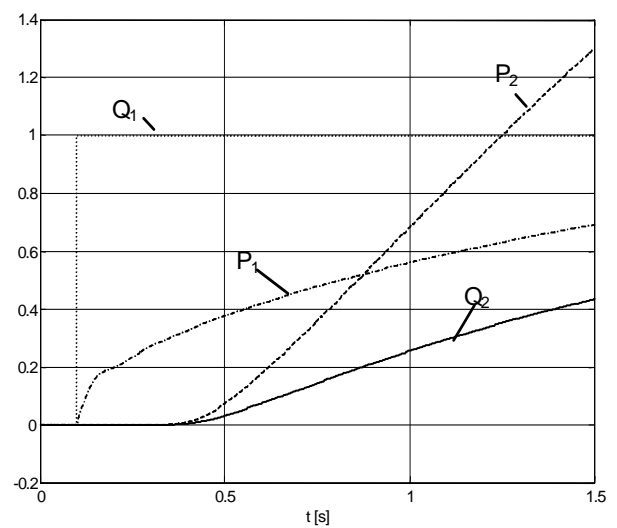

Fig. 18 Transient processes for $V_{g} / V_{t}=\mathbf{0 . 1}$.

where $B_{c}$-bulk modulus of hydraulic pipe, $B_{l}-$ bulk modulus of liquid, $V_{g} / V_{t}$-portion of vapor or gas in total volume, $B_{g}$ - bulk modulus of entrapped gas.

If we assume that $B_{c}=3.45 \times 10^{10} \mathrm{~Pa}, B_{l}=1.4 \times 10^{9}$ $\mathrm{Pa}, B_{g}=2 \times 10^{5} \mathrm{~Pa}$, then transient processes for different gas percentage in the fluid look like in Figs. $16-18(n=8, L s=2 \mathrm{~m})$.

Simulation results show that presence of air in LTL can have great influence on dynamics. Even with small 
volume share of air, character of the transfer process changes completely. Instead of oscillations, we have an aperiodic transfer process and significantly slower system response.

\section{Conclusions}

Model of long transmission line is derived by using the method of pipeline division on final length segments. Inside each of the segments, spatial change is abstracted and only the time changes are observed. Friction, inertia and compressibility are included in the model with use of bond graph elements. Simulation shows that accuracy of results depends on the number of segments the pipeline is approximated. That number depends on the hydraulic circuit where the pipeline is located. Practically, the number of segments should be chosen depending on the frequency of the input signal and dynamics of other elements in the system. It results in obtaining a mathematical model of finite order which can further be reduced to a lower order and still describe the transition process correctly. Besides the pipeline length, effective compressibility of fluid also greatly affects the dynamics. Presence of entrapped vapor or gas slows the transfer process and reduces oscillations in the system.

\section{Acknowledgment}

This work was supported by the Serbian Ministry of
Education and Science under project TR 33026.

\section{References}

[1] D. Matko, G. Geiger, W. Gregoritza, Pipeline simulation techniques, Mathematics and Computers in Simulation 52 (2000) 211-230.

[2] J.S. Stecki, D.C. Davis, Fluid transmission lines-distributed parameter models. Part 1: A review of the state of the art, in: Proceedings of the Institution of Mechanical Engineers. Part A: Journal of Power and Energy, 1986, Vol. 200, No. 4, pp. 215-228.

[3] J.S. Stecki, D.C. Davis, Fluid transmission lines-distributed parameter models. Part 2: Comparison of models, in: Proceedings of the Institution of Mechanical Engineers. Part A: Journal of Power and Energy, 1986, Vol. 200, No. 4, pp. 229-236.

[4] D.A. Hullender, A.J. Healey, Rational polynomial approximations for fluid transmission line models, in: Proceedings of the ASME Winter Annual Meeting-Fluid Transmission Line Dynamics, November 15-20, 1981, Washington, USA, pp. 33-55.

[5] D.A. Hullender, R.L. Woods, C.-H. Hsu, Time domain simulation of fluid transmission lines using minimum order state variable models, in: Proceedings of the ASME Winter Annual Meeting-Fluid Transmission Line Dynamics, November 13-18, 1983, Boston, USA, pp. 78-97.

[6] U. Söderman, Conceptual modelling of physical systems - A frame of reference, Ph.D. Thesis, Linköping University, Linköping, Sweden, 1995.

[7] J. Watton, Fluid Power Systems: Modeling, Simulation, Analog and Microcomputer Control, Prentice Hall International, 1989.

[8] N. Nedić, V. Filipović, Lj. Dubonjić, Design of controllers with fixed order for hydraulic control system with a long transmission line, FME Transactions 38 (2010) 79-86.

[9] H. Merritt, Hydraulic Control Systems, John Wiley \& Sons, 1967. 